\title{
Corneal biomechanics after laser refractive surgery: unmasking differences between techniques
}

Joaquín Fernández, MD, PhD; ${ }^{1,2}$ Manuel Rodríguez-Vallejo, PhD; ${ }^{*}$ Javier Martínez, OD; ${ }^{1}$ Ana Tauste, PhD $;^{1}$ David P Piñero, PhD; ${ }^{2,3}$

${ }^{1}$ Department of Ophthalmology (Qvision), Vithas Virgen del Mar Hospital, 04120, Almería, Spain

2Department of Ophthalmology, Torrecárdenas Hospital Complex, 04009, Almería, Spain ${ }^{3}$ Department of Optics, Pharmacology and Anatomy, University of Alicante, Alicante, Spain ${ }^{4}$ Department of Ophthalmology (OFTALMAR), Vithas Medimar International Hospital, Alicante, Spain

*Corresponding author: manuelrodriguezid@qvision.es (Tel +34686500808)

Declaration of interests

Authors declare no conflict of interest

Submitted: June 15, 2017 


\section{Abstract}

The hypothesis that small incision lenticule extraction provides better preservation of corneal biomechanics than previous laser refractive techniques has led to a growth in the interest on clinical and experimental research in this field. This hypothesis is based on the fact that corneal layers with greater stiffness are preserved with this new technique. However, this hypothesis is controversial as clinical research has shown a great disparity in the outcomes. In this review, we performed an in-depth analysis of the factors that may affect corneal biomechanics in laser refractive surgery procedures from a macro to a microstructural point of view. Differences between laboratory techniques which allow detecting the role of stiffness of thin layers and how a transfer of these improvements is still far from the clinical research are discussed. The macrostructural confounding variables affecting the outcomes obtained with currently available clinical devices are revised and some suggestions for researchers are provided to minimize their influence on measurements. Likewise, less known microstructural confounding variables that are more difficult to control are also described. New advances in algorithms with current devices or the introduction of new devices may help to unmask the possible advantages of small incision lenticule extraction in corneal biomechanics.

\section{Introduction}

Corneal biomechanics has generated great interest among researchers and clinicians in laser refractive surgery (LRS) due to two important factors: postoperative ectasia can occur without apparent preoperative risk factors using the currently available technology, ${ }^{1}$ and the potential influence of biomechanics on the prediction of LRS outcomes. $^{2}$ Corneal ectasia after LRS is the most frightened complication for any 
refractive surgeon. Despite of the wide number of screening tools, including corneal wavefront aberrations, anterior curvature irregularity, thin pachymetry, atypical pachymetry profile, posterior surface elevation anomalies, or epithelial thickness mapping, any of them has shown to be totally effective in screening those corneas with early changes in biomechanical properties associated to the beginning of a ectatic process. ${ }^{3}$ Moreover, new advances have appeared in the last years integrating corneal tomography and dynamic Scheimpflug tonometry in order to increase the sensitivity and specificity for the detection of suspect cases. ${ }^{4}$

The introduction of Small Incision Lenticule Extraction (SMILE) surgery has led to an increase of controversies about corneal biomechanics preservation after laser refractive surgery. Bowman's layer is stiffer than anterior stroma, and in turn, anterior stroma stiffer than posterior. ${ }^{5}$ Since the stiffer layers are much preserved in SMILE, there exists a theoretical basis to hypothesize that this new technique provides a better preservation of corneal biomechanics than LASIK and PRK. ${ }^{6}$ In fact, mathematical biomechanical models have suggested that deeper corrections in the stroma may be possible in SMILE without additional risk for ectasia, because the residual stromal bed supports a greater stress in LASIK than in SMILE. ${ }^{7}$ However, this conclusion has to be interpreted with caution because the link between corneal stress and ectasia has yet to be defined ${ }^{7}$ and a conservative approach similar to that used for LASIK and PRK is being adopted after the report of some ectasias after SMILE in subjects with normal ${ }^{8}$ and suspicious topographies. $^{9-11}$

Despite of the suggestions of mathematical ${ }^{6}$ and finite biomechanical model simulations, ${ }^{7}$ there are not a clear clinical evidence about the SMILE advantage over other LRS techniques in terms of corneal biomechanics. The aims of the current review are the following: (1) to describe the current techniques in laboratory and clinical 
practice for measuring corneal stiffness from the macro to the microstructural point of view and to remark the limitations of these techniques; (2) to identify changes generated in the cornea with different LRS techniques and to understand why biomechanical variations between them are not clinically detected with current clinical devices, and finally (3) to propose some special considerations in the design of clinical studies in order to enhance the capabilities of clinical devices to detect these differences by means of improving the research methodology in order to reduce the impact of confounding variables on the results.

\section{Laboratory versus clinical practice}

The interpretation of results obtained from laboratory methods for assessing corneal biomechanics can be very confusing for the clinician. First, these studies report as results the elastic Young's modulus (YM) and shear moduli obtained from the ratio of applied stress (force) to resultant strain (deformation). However, YM quantifies the response of a perfectly elastic material which is not the case of soft tissues as the cornea. ${ }^{12}$ Therefore, to overcome this problem, soft biological tissues are typically assumed to behave as elastic solids if a significant linear regime of stress-to-strain exists in the limit of small strain response to applied stress. ${ }^{12}$ This allows obtaining the tangent modulus which is defined as the instantaneous slope of the stress-strain curve at a specific stress (Figure 1). ${ }^{13}$

\section{Figure 1}


The resultant YM of the cornea vary widely according to the method of measurement: (1) indentation $(\sim 29 \mathrm{KPa})$, deformation maximized on the point of contact of the indenter depending on the shape of tip used to apply the indentation; (2) tensile stretching ( 3 MPa) which induce macroscopic deformations that span the bulk of a tissue (Figure 1). ${ }^{12}$ It can be stated then that indentation techniques can be optimal to obtain the stiffness of a small location of the tissue from millimeters to nanometers and the tensile techniques to obtain stiffness of the bulk cornea, considering that a higher elastic modulus indicates that the material is stiffer. Furthermore, a limitation of these methods is that the manipulation of the eye for performing the measurement may result in fibre re-orientation, and hence tissue stiffening. A new methodology capable of mechanically testing intact eye globes has been proposed which is based on speckle laser interferometry. The use of this technology has led to conclusions, such as the central cornea is stiffer than the peripheral cornea. ${ }^{14}$

Current techniques for assessing corneal biomechanics in clinical practice are based on the acquisition of some parameters during the corneal deformation by an air-puff. These parameters include the corneal hysteresis $(\mathrm{CH})$ and corneal resistance factor (CRF) derived from the pressures obtained with the Ocular Response Analyzer (ORA; Reichert Ophthalmic Instruments, Depew, NY, USA). More recently, a dynamic Scheimpflug tonometer device (Corvis ST; Oculus Optikgeräte GmbH, Wetzlar, Germany) that records the cornea movement with a high speed camera has been developed that allows the acquisition of multiple parameters including aplannation, times, velocities, deflections, etc. However, parameters obtained with both devices differ widely from the standard descriptors of mechanical properties used in the laboratory. A novel Corneal Indentation Device (CID) has been proposed to measure the tangent modulus of elasticity of the cornea in clinical practice. ${ }^{15-17}$ These three clinical methods are based 
on the response of the bulk cornea and a small change in corneal biomechanics due to the physical composition might be not detected mainly because confounding variables such as corneal thickness and intraocular pressure play a major role in the resistance of the cornea.

\section{The role of corneal layers in corneal biomechanics}

\section{Thin layers of the cornea}

Tensile stretching methods, either pulling a strip of the cornea ${ }^{18}$ or applying a pressure behind the cornea, ${ }^{19}$ have been used to obtain conclusions about the contribution of the different corneal layers to the biomechanics, concluding that some layers might be neglected in numerical simulations due to their small contribution, including the epithelium ${ }^{19,20}$ and the Bowman's layer. ${ }^{18}$ These experiments have been performed measuring the differences in the whole cornea while maintaining or removing the epithelium and Bowman's layer, but without isolating each layer. It is difficult to isolate each layer for mechanical testing as tensile testing requires a mechanical grip to hold and pull the material which would be difficult for thin layers. More sophisticated indentation methods, such as Atomic Force Microscopy (AFM) nano-indentation, have been proposed for a determination of the local elastic modulus of each of the corneal layers. $^{21}$

Last et $\mathrm{al}^{5}$ reported that Bowman's layer has a YM of $109.8 \mathrm{kPa}$, higher than anterior stroma $(33.1 \mathrm{kPa})$ and Descemet membrane $(50 \mathrm{kPa})$. Furthermore, Xia et al. reported an increment of the YM for the stroma ${ }^{22}$ and Descement membrane ${ }^{23}$ from MPa to GPa $\left(10^{9} \mathrm{~Pa}\right)$ with corneal dehydration. From these results, we can hypothesize that the small 
contribution of these layers to the whole corneal biomechanics can be reasonably due to their small thickness in comparison to the total thickness of the cornea. From our perspective, as all these thin layers are affected in keratoconus, ${ }^{24}$ they should not be neglected in the biomechanical future research studies.

The development of advanced imaging techniques, such as serial block face scanning electron microscopy, has enabled to know further about some other micro-structural components as elastic fibers, overlooked in recent years, which differ between normal and keratoconic corneas. ${ }^{25}$ Specifically, it has been reported that cornea contains a network of microfibrils anterior to Descemet's membrane, becoming progressively less abundant anteriorly. Conversely, in the keratoconus cornea elastic fibers are very few anterior to Descemet's membrane and are increased below the basal epithelium in thinned central regions. ${ }^{26}$

\section{The corneal stroma}

The stroma is the thicker layer of the cornea with a microstructural composition of collagen fibrils that varies with depth (Figure 2). The basic structure of collagen is the helical structure of tropocollagen $(1 \mathrm{~nm})$. These molecules are cross-linked to form collagen fibrils $(50-100 \mathrm{~nm})$ and these fibrils are assembled to form collagen fibers or "corneal lamellae" $(500-1000 \mathrm{~nm}) .{ }^{27}$ The stromal thin collagen fibrils are embedded in a soft hydrated matrix formed by proteoglycans and interstitial fluid. ${ }^{28}$ The proteoglycans are composed of protein core and covalently linked glycosaminoglycan side chains and are important for corneal transparency by keeping the fibrils apart at a regular spacing. ${ }^{29}$ Nowadays, it is known that collagen fiber branching density decreases along corneal depth and that the collagen fibers have steep angles in the most anterior part of the cornea with fibers inserted into the Bowman's membrane forming 
bow spring-like structures (Figure 2). ${ }^{30}$ This collagen organization gives a significantly higher stiffness ${ }^{31}$ and less elasticity ${ }^{32}$ to the anterior stroma compared to the middle and posterior stroma.

Furthermore, the anterior cornea is isotropically organized whereas the middle and posterior parts have two preferential orientations attributed to nasal-temporal and inferior-superior directions and the fibers are reorganized with the increase of loading inflation pressure in the middle and posterior stroma. ${ }^{33}$ The stiffness of the stroma varies with depth from $7.71 \mathrm{Kpa}$ to $240 \mathrm{Kpa}$ for the anterior stroma, from $1.99 \mathrm{Kpa}$ to $70 \mathrm{Kpa}$ for the central stroma and from $1.31 \mathrm{Kpa}$ to $10 \mathrm{Kpa}$ for the posterior stroma. ${ }^{34,35}$

\section{Figure 2}

\section{Macro and microstructural changes after surgery}

From a theoretical perspective, SMILE preserves the stiffer layers of the cornea and for eyes with the same intraocular pressure and the same corneal thickness after surgery corneas should be stiffer after SMILE than after PRK or LASIK. However, some other microstructural changes can lead to variations in the stiffness among techniques or even for the same technique. Knox et al reported greater alteration of mechanical properties increasing the LASIK flap depth from 90 to $160 \mu \mathrm{m}$, and less variation for horizontal delaminations in comparison to vertical side-cuts. ${ }^{20}$ However, the advantages of horizontal delaminations should be interpreted with caution due to the possibility of increasing the risk of epithelial ingrowth. ${ }^{36}$ Wang et al. reported that the creation of a thin flap of $90-110 \mu \mathrm{m}$ in rabbits did not additionally compromise the elastic modulus of the cornea and the mechanical response of the cornea after LASIK may be 
predominantly influenced by laser ablation. ${ }^{37} \mathrm{He}$ et al. reported a higher YM in rabbits operated on with the SMILE technique using a lenticule thickness of $160 \mu \mathrm{m}$ compared to the use of $100 \mu \mathrm{m} .{ }^{38}$ Likewise, Spiru et $\mathrm{al}^{39}$ found that the YM after SMILE treatment was significantly higher than after FLEx in ex-vivo porcine corneas, suggesting that cap-based technique SMILE can be considered superior in terms of biomechanical stability.

Santhiago et al reported that the percentage of tissue altered, including flap thickness and ablation depth, is a more robust risk factor for corneal ectasia than residual stromal bed and central corneal thickness. ${ }^{40}$ They suggested that for subjects with the same percentage of tissue ablated, a major risk of corneal ectasia can be presented in those with thicker flaps than in those with greater ablation depth. ${ }^{40}$ Kling reported no differences in the biomechanical properties measured with stress-strain extensometry in porcine corneas after retreatment with SMILE and PRK in comparison to non-treated corneas, but less elastic modulus was found after LASIK than in the control group. ${ }^{41}$ Despite a highest preservation of corneal biomechanics was expected after SMILE compared to PRK, no differences were found that may be due in part to the fact that Bowman's membrane in porcine corneas is not as highly developed as in humans or primates. $^{41}$

The epithelium thickness is increased after laser refractive surgery in comparison with non-operated eyes. In SMILE, this increment has been reported to be between 2.51 $\mu \mathrm{m}^{42}$ and $15 \mu \mathrm{m}^{43}$ greater at the center, decreasing along the periphery and acquiring a lenticular shape ${ }^{44}$ Furthermore, the central epithelium hyperplasia has been reported to be less after SMILE than after LASIK (around $1 \mu \mathrm{m}$ ). ${ }^{42}$ This epithelial remodeling in SMILE has been reported to be correlated with age and the corrected refractive error but not with the refractive results. ${ }^{45}$ Conversely, the difference between the achieved 
stromal reduction in comparison to the planned tissue removal has been reported to be between $8 \mu \mathrm{m}^{43}$ and $11.9 \mu \mathrm{m}$ thicker on average for SMILE but only $0.4 \mu \mathrm{m}$ for LASIK at 3 months postoperatively, which was related with the residual refractive error. ${ }^{43,42}$ From these findings, it has been hypothesized that there exists an stromal expansion after SMILE $^{43}$ that is compensated with a less increase of the central epithelial thickness. ${ }^{42}$ This can led us to hypothesize that corneas with the same thickness after surgery might be stiffer in SMILE than LASIK due to the expansion of the stroma instead of the epithelium which is a less stiff layer. ${ }^{19,20}$ However, this is only an hypothesis that should be confirmed in future studies because the nature of this expansion may vary the stiffness in the stroma in comparison to the untreated cornea.

Corneal hydration also has an important role on corneal biomechanics. It is important to note that as the permeability of the cornea increases, the cornea becomes thicker but the stiffness decreases from GPa to MPa. ${ }^{22,46}$ This means that two corneas with the same thickness might have different biomechanical behavior depending on the level of corneal hydration. Regarding this topic, it would be interesting to differentiate between the components from stromal collagen fibrils and the soft hydrated matrix. ${ }^{28}$ The combination of corneal densitometry and biomechanical analysis may be a possible option to characterize the impact of corneal hydration. ${ }^{47}$

\section{The combination of densitometry and biomechanics}

The corneal structure previously described leads to disparities in the refractive index (RI) which produce light scattering visible through densitometry maps. ${ }^{48}$ The major sources of light scattering are the anterior superficial corneal epithelial cell layer and the posterior corneal endothelium because of the higher difference in RI from air and 
aquous. ${ }^{49}$ However, variations of light scattering are also presented within the cornea due to disparities of RI along epithelium, anterior stroma and posterior stroma. ${ }^{50}$ Furthermore, different RIs are presented in the hydrated fibrils and the extrafibrilar matrix, and the RI of the corneal stroma is reduced as the cornea swells. ${ }^{51}$ In fact, the RI increment of the stroma has been associated with the dehydration after LASIK, ${ }^{52}$ and it is higher in older corneas which directly correlates with the increase of densitometry ${ }^{53}$ and stiffness. ${ }^{54}$

At the baseline state, the densitometry measured with the Pentacam system (Oculus) is highest in the anterior $120 \mu \mathrm{m}$ of the cornea in comparison to the center and posterior layers. ${ }^{53}$ Considering that the normal epithelial thickness is around $53.4 \pm 4.6 \mu \mathrm{m}^{55}$ and that the lamellar angles relative to the stromal surface are highest in the anterior-most 83 $\mu \mathrm{m}$ of the corneal stroma, our hypothesis is that the increase of the anterior densitometry is not only due to epithelial thickness but also to the angle of the collagen lamellae ${ }^{30}$ at this part of the cornea and possibly due to the fact that the anterior stroma tends to be less hydrated and more resistant to water flow than the posterior stroma. ${ }^{52}$

The densitometry increases after laser refractive surgery, ${ }^{56}$ probably due to the increase of the RI because of stromal dehydration by the laser application, ${ }^{52}$ and returns to values ever below the preoperative status at 12 month after PRK ${ }^{56}$ and SMILE ${ }^{57}$ Furthermore, the preoperative values are reached at 3 months after LASIK and SMILE without differences between techniques, ${ }^{58}$ but not after PRK. ${ }^{56}$ The decrease in densitometry over the postoperative time period might be related to the recovery of corneal hydration, with a decrease in the refractive index of the stroma, ever for levels of hydration higher than preoperative values. This is in an agreement with the possible expansion of the stroma and the increment of corneal thickness with time..$^{59,60}$ 
We reported for the first time the potential usefulness of dynamic densitometry in refractive surgery. It is defined as the increase in densitometry during the course of the air-puff generated with the Corvis ST system (Oculus). It is important to differentiate between static densitometry measured with any rotating Scheimpflug camera and the dynamic densitometry measured with the Corvis ST system. The first one represents the natural state of the corneal fibrils and corneal hydration whereas the latter hypothetically would represent the modification of collagen fibers order and fluidics movement along the cornea during the air-puff course. We found that densitometry was increased during the inward stage, reaching the maximum value close to the highest concavity, whereas during the outward stage the densitometry at the second applanation status was higher than that obtained at first applanation. ${ }^{47}$ Our explanation about the course of dynamic densitometry is that the stromal fluid goes from the anterior to the posterior stroma with the air puff pressure, whilst the anterior fibers are compressed or reordered. Furthermore, we reported that the densitometry sign described as a brightness inclined fringe that appears in the peripheral corneal peaks at the highest concavity stage, moving to the corneal periphery until its disappearance, has a higher prevalence after SMILE. Specifically, the prevalence changed from $48.8 \%$ preoperatively to $72.1 \%$ postoperatively. This sign might be related with a greater fluid movement due to the alteration of collagen fibers during surgery. ${ }^{47}$ However, this is only an hypothesis that suggests the possible advantages of including corneal densitometry in the algorithms to compute corneal stiffness. 


\section{Evidence on corneal biomechanical changes in the comparison of refractive surgery techniques}

Table 1 includes a summary with the main conclusions obtained from different studies that have measured corneal biomechanics in different LRS techniques, either with the ORA or the Corvis ST systems. Three of four studies agreed that LASIK/FS-LASIK have more affectation of the biomechanical parameters provided by those devices than PRK/LASEK. In contrast, differences between SMILE and FS-LASIK were more controversial, with no study supporting the hypothesis that FS-LASIK leads to fewer changes in biomechanical parameters compared to SMILE. The controversy increases in the comparison of SMILE and PRK/LASEK, without any tendency in favor of one of both techniques. Some other particularities with regard studies performed with variants of the same technique are also described in Table 1.

\section{Table 1}

\section{Minimizing confounding variables with clinical devices}

The response of the cornea to an indentation load, similar to the air-puff of dynamic Scheimpflug tonometry, depends on the physical composition of the cornea, the intraocular pressure, and the corneal thickness. ${ }^{83}$ To enhance the ability of clinical devices to detect differences between LRS techniques, it is essential to minimize the possible effect of corneal thickness and intraocular pressure on the biomechanical measurements provided. However, despite of the performance of good and well-design studies for minimizing the impact of confounding variables, such as intraocular pressure and corneal thickness, it is still unclear if differences in the biomechanical properties after SMILE measured by dynamic Scheimpflug tonometry could be biased because the 
anterior collagen fibers during the loading of the air-puff are submitted to compression instead of tension stress. ${ }^{83}$

Moreover, for continue advancing in the clinical research with the currently available devices, some basic considerations should be considered if biomechanical changes are intended to be evaluated after LRS with different techniques:

1. Subjects should be measured preoperatively and postoperatively in the same timetable range. Although Hon et $\mathrm{al}^{13}$ reported no differences in tangent modulus with the CID due to the variation of IOP and CCT during the wake time, Ariza et $\mathrm{al}^{84}$ suggested that with air-puff devices, corneal displacement variations can reach 5\% during the day depending on the level of stiffness of the cornea evaluated, with less variability from 10:00 AM to 13:00 $\mathrm{PM}^{84}$ Indeed, we reported that the biomechanically corrected intraocular pressure of Corvis ST predicted better the preoperative IOP in subjects operated on with SMILE when the analysis was applied considering only subjects measured in the same time slot preoperatively and postoperatively. ${ }^{47}$

2. Biomechanical parameters should be compared considering their relative change as a function of the removed corneal thickness, especially when using parameters that have been demonstrated to be significantly correlated with corneal thickness. ${ }^{82}$ Tissue removal in LASIK may be approximately determined with the Munnerlyn formula, ${ }^{85}$ but in SMILE a thickness of $10-15 \mu \mathrm{m}$ should be added corresponding to the edge thickness. Therefore, there is a slightly higher tissue removal in SMILE due to this and due to the quadratic dependency on the diameter of the optical zone. ${ }^{86}$ The real removed thickness should be then considered instead of the refractive error treated. We reported that the new stiffness parameter ${ }^{87}$ and biomechanically corrected intraocular pressure of 
Corvis ST were the only two parameters for which this correlation with removed corneal thickness was not presented. ${ }^{47}$

3. Exclusion criteria should include any subject under treatments that may have any influence on corneal thickness or intraocular pressure due to its possible impact on corneal composition and then on corneal biomechanics. ${ }^{88}$

\section{Conclusions}

In this review, we have conducted an exhaustive analysis of the corneal structure and methods for measuring corneal stiffness in order to understand the role of corneal composition on biomechanics. The stiffness highly depends on the measurement method used in the laboratory, with a great variation between studies that may difficult the incorporation of this information in biomechanical models. The role of corneal layers should be considered as the stiffness relative to the thickness or, in other words, how stiff is the layer considering its thickness in comparison to the total cornea. Under this scenario, Bowman's and Descemet's layers are stiffer than the anterior stroma and this greater stiffness is masked when we use methods that evaluate the stiffness of the bulk cornea. Currently available clinical methods to characterize corneal biomechanics are not able to assess the stiffness of each of the layers of the cornea and the only possibility is to measure the bulk cornea. This may be considered as a limitation because of the impact of confounding variables, such as corneal thickness and intraocular pressure. According to the peer-reviewed literature, we have defined some considerations to minimize the influence of these confounding variables on the results. Despite of applying this consideration in future studies, we cannot ensure that it will allow clinicians to clarify if SMILE is superior in terms of corneal biomechanics to 
other LRS techniques. With the currently available clinical devices, as there are microstructural changes as corneal hydration alterations, stromal expansion or anterior stromal compression during the loading stage of the air puff, these may also act as confounding variables. Regarding the more recent advances on corneal biomechanics, it should be remarked that the new parameters of the Corvis ST system are not correlated with corneal thickness and dynamic corneal densitometry may be an additional tool in algorithms characterizing corneal biomechanics with the currently available devices.

\section{References}

1. Klein SR, Epstein RJ, Randleman JB, Stulting RD. Corneal ectasia after laser in situ keratomileusis in patients without apparent preoperative risk factors. Cornea. 2006;25(4):388-403.

2. Roy AS, Dupps WJ. Effects of altered corneal stiffness on native and postoperative LASIK corneal biomechanical behavior: A whole-eye finite element analysis. $J$ Refract Surg. 2009;25(10):875-887.

3. Roberts CJ, Dupps WJ. Biomechanics of corneal ectasia and biomechanical treatments. J Cataract Refract Surg. 2014;40(6):991-998.

4. Vinciguerra R, Ambrósio R, Elsheikh A, et al. Detection of keratoconus with a new biomechanical index. J Refract Surg. 2016;32(12):803-810.

5. Last JA, Thomasy SM, Croasdale CR, Russell P, Murphy CJ. Compliance profile of the human cornea as measured by atomic force microscopy. Micron. 2012;43(12):12931298.

6. Reinstein DZ, Archer TJ, Randleman JB. Mathematical model to compare the relative tensile strength of the cornea after PRK, LASIK, and small incision lenticule extraction. J Refract Surg. 2013;29(7):454-460.

7. Sinha Roy A, Dupps WJ, Roberts CJ. Comparison of biomechanical effects of smallincision lenticule extraction and laser in situ keratomileusis: Finite-element analysis. J Cataract Refract Surg. 2014;40(6):971-980.

8. Sachdev G. Unilateral corneal ectasia following small-incision lenticule extraction. $J$ Cataract Refract Surg. 2015;41(9):2014-2018.

9. Wang $\mathrm{Y}$, Cui C, Li Z, et al. Corneal ectasia 6.5 months after small-incision lenticule extraction. J Cataract Refract Surg. 2015; 41(5):1100-6

10. El-Naggar MT. Bilateral ectasia after femtosecond laser-assisted small-incision lenticule extraction. J Cataract Refract Surg. 2015;41(4):884-888.

11. Mattila JS, Holopainen JM. Bilateral ectasia after femtosecond laser-assisted small incision lenticule extraction (SMILE). J Refract Surg. 2016;32(7):497-500. 
12. McKee CT, Last JA, Russell P, Murphy CJ. Indentation versus tensile measurements of Young's modulus for soft biological tissues. Tissue Eng Part B Rev. 2011;17(3):155-164.

13. Hon Y, Wan K, Chen G-Z, Lu S-H, Lam DCC, Lam AKC. Diurnal variation of corneal tangent modulus in Normal Chinese. Cornea. 2016;35(12):1600-1604.

14. De la Torre IM, Hernández Montes M del S, Flores-Moreno JM, Santoyo FM. Laser speckle based digital optical methods in structural mechanics: A review. Opt Lasers Eng. 2016;(February):1-27.

15. Hon Y, Chen G-Z, Lu S-H, Lam DCC, Lam AKC. High myopes have lower normalised corneal tangent moduli (less "stiff" corneas) than low myopes. Ophthalmic Physiol Opt. 2017;37(1):42-50.

16. Lam AKC, Hon Y, Leung LKK, Lam DCC. Repeatability of a novel corneal indentation device for corneal biomechanical measurement. Ophthalmic Physiol Opt. 2015;35(4):455-461.

17. Ko MWL, Leung LKK, Lam DCC, Leung CKS. Characterization of corneal tangent modulus in vivo. Acta Ophthalmol. 2013;91(4):263-269.

18. Seiler T, Matallana M, Sendler S, Bende T. Does Bowman's layer determine the biomechanical properties of the cornea? Refract Corneal Surg. 8(2):139-142.

19. Elsheikh A, Alhasso D, Rama P. Assessment of the epithelium's contribution to corneal biomechanics. Exp Eye Res. 2008;86(2):445-451.

20. Knox Cartwright NE, Tyrer JR, Jaycock PD, Marshall J. Effects of variation in depth and side cut Angulations in LASIK and thin-flap LASIK using a femtosecond laser: A Biomechanical Study. J Refract Surg. 2012;28(6):419-425.

21. Lombardo M, Lombardo G, Carbone G, de Santo MP, Barberi R, Serrao S. Biomechanics of the anterior human corneal tissue investigated with atomic force microscopy. Investig Ophthalmol Vis Sci. 2012;53(2):1050-1057.

22. Xia $D$, Zhang $S$, Hjortdal $\rfloor \varnothing$, et al. Hydrated human corneal stroma revealed by quantitative dynamic atomic force microscopy at nanoscale. ACS Nano. 2014;8(7):68736882.

23. Xia D, Zhang $\mathrm{S}$, Nielsen $\mathrm{E}$, et al. The ultrastructures and mechanical properties of the descement's membrane in fuchs endothelial corneal dystrophy. Sci Rep. 2016;16(6):23096.

24. Khaled ML, Helwa I, Drewry M, Seremwe M, Estes A, Liu Y. Molecular and Histopathological Changes Associated with Keratoconus. Biomed Res Int. 2017; 2017:7803029.

25. Lewis PN, White TL, Young RD, Bell JS, Winlove CP, Meek KM. Three-dimensional arrangement of elastic fibers in the human corneal stroma. Exp Eye Res. 2016;146:4353.

26. White TL, Lewis PN, Young RD, et al. Elastic microfibril distribution in the cornea: Differences between normal and keratoconic stroma. Exp Eye Res. 2017;159:40-48.

27. Bueno JM, Ávila FJ, Artal P. Chapter 5. Second harmonic generation microscopy : A tool for quantitative analysis of tissues. In: ed. Stefan G. Stanciu. Microscopy and Analysis; In Tech. 2016:99-119. Available from: https://www.intechopen.com/books/microscopyand-analysis/second-harmonic-generation-microscopy-a-tool-for-quantitative-analysis- 
of-tissues. Accessed: 1/23/2018.

28. Hatami-Marbini H, Etebu E. An experimental and theoretical analysis of unconfined compression of corneal stroma. J Biomech. 2013;46(10):1752-1758.

29. Jastrzebska $M$, Tarnawska $D$, Wrzalik $R$, et al. New insight into the shortening of the collagen fibril D-period in human cornea. J Biomol Struct Dyn. 2017; 35(3):551-563

30. Abass A, Hayes S, White N, Sorensen T, Meek KM. Transverse depth-dependent changes in corneal collagen lamellar orientation and distribution. $J R$ Soc Interface. 2015;12(104):20140717

31. Quantock AJ, Winkler M, Parfitt GJ, et al. From nano to macro: Studying the hierarchical structure of the corneal extracellular matrix. Exp Eye Res. 2015;133:81-99.

32. Janice Dias, Ziebarth NM. Anterior and posterior corneal stroma elasticity assessed using nanoindentation. Exp Eye Res. 2013;29(6):997-1003.

33. Benoit A, Latour G, Allain J. Simultaneous microstructural and mechanical characterization of human corneas at increasing pressure. J Mech Behav Biomed Mater. 2016: 60:93-105.

34. Sloan SR, Khalifa YM, Buckley MR. The location- and depth-dependent mechanical response of the human cornea under shear loading. Invest Ophthalmol Vis Sci. 2014;55(12):7919-7924.

35. Petsche SJ, Chernyak D, Martiz J, Levenston ME, Pinsky PM. Depth-dependent transverse shear properties of the human corneal stroma. Investig Ophthalmol Vis Sci. 2012;53(2):873-880.

36. Jhanji V, Chan TCY, Li WY, et al. Conventional versus inverted side-cut flaps for femtosecond laser-assisted LASIK: Laboratory and clinical evaluation. J Refract Surg. 2017;33(2):96-103.

37. Wang X, Li X, Chen W, He R, Gao Z, Feng P. Effects of ablation depth and repair time on the corneal elastic modulus after laser in situ keratomileusis. Biomed Eng Online. 2017;16(1):20.

38. He $M$, Wang $W$, Ding $H$, Zhong $X$. Comparison of two cap thickness in small incision lenticule extraction: $100 \mu \mathrm{m}$ versus 160 $\mu \mathrm{m}$. PLoS One. 2016;11(9):e0163259.

39. Spiru B, Kling S, Hafezi F, Sekundo W. Biomechanical differences between femtosecond lenticule extraction (FLEx) and small incision lenticule extraction (SmILE) tested by 2Dextensometry in ex vivo porcine eyes. Investig Opthalmology Vis Sci. 2017;58(5):2591.

40. Santhiago MR, Smajda D, Wilson SE, Randleman JB. Relative contribution of flap thickness and ablation depth to the percentage of tissue altered in ectasia after laser in situ keratomileusis. J Cataract Refract Surg. 2015;41(11):2493-2500.

41. Kling S, Spiru B, Hafezi F, Sekundo W. Biomechanical weakening of different retreatment options after small incision lenticule extraction (SMILE). J Refract Surg. 2017;33(3):193-198.

42. Ryu I-H, Kim BJ, Lee J-H, Kim SW. Comparison of corneal epithelial remodeling after femtosecond laser-assisted LASIK and small incision lenticule extraction (SMILE). J Refract Surg. 2017;33(4):250-256.

43. Reinstein DZ, Archer TJ, Gobbe M. Lenticule thickness readout for small incision 
lenticule extraction compared to Artemis three-dimensional very high-frequency digital ultrasound stromal measurements. J Refract Surg. 2014; 30(5):304-9.

44. Luft N, Ring MH, Dirisamer M, et al. Semiautomated SD-OCT measurements of corneal sublayer thickness in normal and post-SMILE eyes. Cornea. 2016; 35(7):972-9.

45. Luft $\mathrm{N}$, Ring $\mathrm{MH}$, Dirisamer $\mathrm{M}$, et al. Corneal epithelial remodeling induced by small incision lenticule extraction (SMILE). Investig Ophthalmol Vis Sci. 2016;57(9):OCT176183.

46. Hatami-Marbini H, Etebu E. Hydration dependent biomechanical properties of the corneal stroma. Exp Eye Res. 2013;116:47-54.

47. Fernandez J, Rodríguez-Vallejo M, Martínez J, Tauste A, Salvestrini P, Piñero DP. New parameters for evaluating corneal biomechanics and intraocular pressure after SMILE by Scheimpflug-Based Dynamic Tonometry. J Cataract Refract Surg. 2017: 43(6):803811.

48. Cook CA, Koretz JF. Methods to obtain quantitative parametric descriptions of the optical surfaces of the human crystalline lens from Scheimpflug slit-lamp images. I. Image processing methods. J Opt Soc Am A Opt Image Sci Vis. 1998;15(6):1473-1485.

49. Otri AM, Fares U, Al-Aqaba MA, Dua HS. Corneal densitometry as an indicator of corneal health. Ophthalmology. 2012;119(3):501-508.

50. Patel S, Marshall J, Fitzke FW. Refractive index of the human corneal epithelium and stroma. J Refract Surg. 1995;11(2):100-105.

51. Meek KM, Dennis S, Khan S. Changes in the refractive index of the stroma and its extrafibrillar matrix when the cornea swells. Biophys J. 2003;85(4):2205-2212.

52. Patel S, Alió JL, Pérez-Santonja JJ. Refractive index change in bovine and human corneal stroma before and after LASIK: A study of untreated and re-treated corneas implicating stromal hydration. Investig Ophthalmol Vis Sci. 2004;45(10):3523-3530.

53. Ní Dhubhghaill S, Rozema JJ, Jongenelen S, Ruiz Hidalgo I, Zakaria N, Tassignon MJ. Normative values for corneal densitometry analysis by scheimpflug optical assessment. Inves Opthal Vis Sci. 2014;55(1):162.

54. Elsheikh A, Wang D, Brown M, Rama P, Campanelli M, Pye D. Assessment of corneal biomechanical properties and their variation with age. Curr Eye Res. 2007;32(1):11-19.

55. Reinstein DZ, Archer TJ, Gobbe M, Silverman RH, Coleman DJ. Epithelial thickness in the normal cornea: three-dimensional display with very high frequency ultrasound. $J$ Refract Surg. 2008;24(6):571-581.

56. Cennamo G, Forte R, Aufiero B, La Rana A. Computerized Scheimpflug densitometry as a measure of corneal optical density after excimer laser refractive surgery in myopic eyes. J Cataract Refract Surg. 2011;37(8):1502-1506.

57. Pedersen IB, Ivarsen A, Hjortdal J. Changes in astigmatism, densitometry, and aberrations after SMILE for low to high myopic astigmatism: A 12-month prospective Study. J Refract Surg. 2017;33(1):11-17.

58. Lazaridis A, Droutsas K, Sekundo W, Petrak M, Schulze S. Clinical study corneal clarity and visual outcomes after Small-Incision Lenticule Extraction and comparison to Femtosecond Laser-Assisted In Situ Keratomileusis. J Ophthalmol. 2017;2017: 5646390. 
59. Ivarsen A, Fledelius W, Hjortdal JØ. Three-year changes in epithelial and stromal thickness after PRK or LASIK for high myopia. Investig Ophthalmol Vis Sci. 2009;50(5):2061-2066.

60. Ivarsen A, Hjortdal J. Seven-year changes in corneal power and aberrations after PRK or LASIK. Investig Ophthalmol Vis Sci. 2012;53(10):6011-6016.

61. Kirwan C, O'Keefe M. Corneal hysteresis using the Reichert ocular response analyser: findings pre- and post-LASIK and LASEK. Acta Ophthalmol. 2008;86(2):215-218.

62. Kamiya K, Shimizu K, Ohmoto F. Comparison of the changes in corneal biomechanical properties after photorefractive keratectomy and laser in situ keratomileusis. Cornea. 2009;28(7):765-769.

63. Hassan Z, Modis L, Szalai E, Berta A, Nemeth G. Examination of ocular biomechanics with a new Scheimpflug technology after corneal refractive surgery. Cont Lens Anterior Eye. 2014;37(5):337-341.

64. Shen Y, Chen Z, Knorz M, Li M, Zhao J, X Zhou. Comparison of corneal deformation parameters after SMILE, LASEK, and femtosecond laser-assisted LASIK. J Refract Surg. 2014;30(5):310-318.

65. Pedersen IB, Bak-Nielsen S, Vestergaard AH, Ivarsen A, Hjortdal J. Corneal biomechanical properties after LASIK, ReLEx flex, and ReLEx smile by Scheimpflug-based dynamic tonometry. Graefes Arch Clin Exp Ophthalmol. 2014;252(8):1329-1335.

66. Wang $\mathrm{D}$, Liu $\mathrm{M}$, Chen $\mathrm{Y}$, et al. Differences in the corneal biomechanical changes after SMILE and LASIK. J Refract Surg. 2014;30(10):702-707.

67. Wu D, Wang Y, Zhang L, Wei S, Tang X. Corneal biomechanical effects: small-Incision lenticule extraction versus femtosecond laser-assisted laser in situ keratomileusis. J Cataract Refract Surg. 2014;40(6):954-962.

68. Agca A, Ozgurhan EB, Demirok A, et al. Comparison of corneal hysteresis and corneal resistance factor after small incision lenticule extraction and femtosecond laser-assisted LASIK: A prospective fellow eye study. Cont Lens Anterior Eye. 2014;37(2):77-80.

69. Sefat SMM, Wiltfang R, Bechmann M, Mayer WJ, Kampik A, Kook D. Evaluation of changes in human corneas after femtosecond laser-assisted LASIK and small-incision lenticule extraction (SMILE) using non-contact tonometry and ultra-high-speed camera (Corvis ST). Curr Eye Res. 2016;41(7):917-922.

70. Osman IM, Helaly HA, Abdalla M, Shousha MA. Corneal biomechanical changes in eyes with small incision lenticule extraction and laser assisted in situ keratomileusis. $B M C$ Ophthalmol. 2016;16(1):123.

71. Wang B, Zhang Z, Naidu RK, et al. Comparison of the change in posterior corneal elevation and corneal biomechanical parameters after small incision lenticule extraction and femtosecond laser-assisted LASIK for high myopia correction. Contact Lens Anterior Eye. 2016; 39(3):191-6.

72. Zhang J, Zheng $\mathrm{L}$, Zhao $\mathrm{X}, \mathrm{Xu} \mathrm{Y}, \mathrm{Chen} \mathrm{S}$. Corneal biomechanics after small-incision lenticule extraction versus $Q$-value-guided femtosecond laser-assisted in situ keratomileusis. J Curr Ophthalmol. 2016;28(4):181-187.

73. Dou R, Wang Y, Xu L, Wu D, Wu W, Li X. Comparison of corneal biomechanical characteristics after surface ablation refractive surgery and novel lamellar refractive 
surgery. Cornea. 2015;34(11):1441-1446.

74. Yıldırım Y, Ölçücü O, Başcı A, et al. Comparison of Changes in Corneal Biomechanical Properties after Photorefractive Keratectomy and Small Incision Lenticule Extraction. Türk Oftalmol Derg. 2016;46(2):47-51.

75. Chen M, Yu M, Dai J. Comparison of biomechanical effects of small incision lenticule extraction and laser-assisted subepithelial keratomileusis. Acta Ophthalmol. 2016;94(7):e586-e591.

76. Al-Nashar $\mathrm{H}, \mathrm{Awad} \mathrm{A}$. Comparison of corneal hysteresis and corneal resistance factor after small-incision lenticule extraction and photorefractive keratectomy. Delta J Ophthalmol. 2017;18(1):1.

77. Kamiya K, Shimizu K, Igarashi A, Kobashi H, Sato N, Ishii R. Intraindividual comparison of changes in corneal biomechanical parameters after femtosecond lenticule extraction and small-incision lenticule extraction. J Cataract Refract Surg. 2014;40(6):963-970.

78. Shen $\mathrm{Y}$, Zhao J, Yao $\mathrm{P}$, et al. Changes in corneal deformation parameters after lenticule creation and extraction during small incision lenticule extraction (SMILE) procedure. PLoS One. 2014;9(8):e103893.

79. Mastropasqua L, Calienno R, Lanzini M, et al. Evaluation of corneal biomechanical properties modification after small incision lenticule extraction using Scheimpflugbased noncontact tonometer. Biomed Res Int. 2014;2014:290619.

80. El-Massry AA, Goweida MB, Shama Ael-S, Elkhawaga MH, Abdalla MF. Contralateral eye comparison between femtosecond small incision intrastromal lenticule extraction at depths of 100 and $160 \mu \mathrm{m}$. Cornea. 2015;34(10):1272-1275.

81. Leccisotti A, Fields SV, Moore J, Shah S, Moore TC. Changes in ocular biomechanics after femtosecond laser creation of a laser in situ keratomileusis flap. J Cataract Refract Surg. 2016;42(1):127-131.

82. Fernández J, Rodríguez-Vallejo M, Martínez J, Tauste A, Piñero DP. Corneal thickness after SMILE affects scheimpflug-based dynamic tonometry. J Refract Surg. 2016;32(12):821-828.

83. Ariza-Gracia MA, Zurita JF, Piñero DP, Rodriguez-Matas JF, Calvo B. Coupled biomechanical response of the cornea assessed by non-contact tonometry. A simulation study. PLoS One. 2015;10(3):e0121486.

84. Ariza-Gracia MA, Piñero DP, Rodriguez JF, Pérez-Cambrodí RJ, Calvo B. Interaction between diurnal variations of intraocular pressure, pachymetry, and corneal response to an air puff: Preliminary evidence. JCRS Online Case Reports. 2014;3(1):12-15.

85. Chang AW, Tsang AC, Contreras JE, et al. Corneal tissue ablation depth and the Munnerlyn formula. J Cataract Refract Surg. 2003;29(6):1204-1210.

86. Bischoff $M$, Strobrawa G. Femtosecond laser keratomes for small incision lenticule extraction (SMILE). In: Sekundo W, ed. Small Incision Lenticule Extraction (SMILE): Principles, Techniques, Complication Management, and Future Concepts. Springer. London; 2015:9.

87. Roberts CJ, Mahmoud AM, Bons JP, et al. Introduction of two novel stiffness parameters and interpretation of air puff-induced biomechanical deformation parameters with a dynamic scheimpflug analyzer. J Refract Surg. 2017;33(4):266-273. 
88. Alnawaiseh $\mathrm{M}$, Zumhagen L, Zumhagen $\mathrm{S}$, et al. Corneal densitometry as a novel technique for monitoring amiodarone therapy. Ophthalmology. 2016;123(11):22942299.

\section{Figure captions}

Figure 1. Graphical representation of a non-linear stress/strain function typical from the cornea. Tangent modulus is obtained from the slope at a particular stress for which the function is almost linear (elastic behavior). A graphical description of tensile stretching is included in the figure to explain the stress/strain relationship.

Figure 2. Scheme for the role of different variables in the clinical measure of corneal biomechanics. From the macro to the micro structural point of view, the role of the different variables decreases in comparison to the mechanical displacement of the bulk cornea. 
Table 1. Studies analyzing corneal biomechanics after laser refractive surgery techniques

\begin{tabular}{|c|c|c|c|}
\hline Authors & Instruments & Techniques & Conclusions \\
\hline \multicolumn{4}{|l|}{ LASIK vs LASEK / PRK } \\
\hline$(\text { Kirwan et al. 2008) })^{61}$ & ORA & LASIK, LASEK & No differences among techniques \\
\hline$(\text { Kamiya et al. 2009) })^{62}$ & ORA & LASIK, PRK & More affectation after LASIK \\
\hline$\left(\right.$ Hassan et al. 2014) ${ }^{63}$ & CORVIS & LASIK, PRK & More affectation after LASIK \\
\hline (Shen et al. 2014)64 & CORVIS & FS-LASIK, LASEK & More affectation after FS-LASIK \\
\hline \multicolumn{4}{|l|}{ SMILE vS LASIK } \\
\hline$(\text { Pedersen et al. 2014) })^{65}$ & ORA; CORVIS & SMILE, FS-LASIK, & No differences among techniques \\
\hline (Wang et al. 2014) $^{66}$ & ORA & SMILE, FS-LASIK & $\begin{array}{l}\text { Differences for myopias of more than } \\
6 \text { D favorable to SMILE }\end{array}$ \\
\hline$\left(\right.$ Shen et al. 2014) ${ }^{64}$ & CORVIS & SMILE, FS-LASIK, & No differences among techniques \\
\hline$(\mathrm{Wu} \text { et al. 2014) })^{67}$ & ORA & SMILE, FS-LASIK & More affectation after FS-LASIK \\
\hline$(\text { Agca et al. 2014) })^{68}$ & ORA & SMILE, FS-LASIK & No differences among techniques \\
\hline$(\text { Sefat et al. 2016) })^{69}$ & CORVIS & SMILE, FS-LASIK & No differences among techniques \\
\hline$($ Osman et al. 2016) & ORA & SMILE, FS-LASIK & More affectation after FS-LASIK \\
\hline (Wang et al. 2016) ${ }^{71}$ & ORA & SMILE, FS-LASIK & More affectation after FS-LASIK \\
\hline (Zhang et al. 2016) ${ }^{72}$ & ORA & $\begin{array}{l}\text { SMILE, FS-LASIK } \\
\text { WF }\end{array}$ & No differences among techniques \\
\hline \multicolumn{4}{|l|}{ SMILE vs LASEK/PRK } \\
\hline$(\text { Shen et al. 2014) })^{64}$ & CORVIS & SMILE, LASEK & No differences among techniques \\
\hline (Dou et al. 2015) ${ }^{73}$ & ORA & SMILE, LASEK & $\begin{array}{l}\text { In terms of per unit tissue removed, } \\
\text { SMILE seems to have less effect on } \\
\text { corneal biomechanics than LASEK }\end{array}$ \\
\hline (Y1ldırım et al. 2016) & ORA & SMILE; PRK & More affectation after SMILE \\
\hline$(\text { Chen et al. 2016) })^{75}$ & ORA & SMILE; LASEK & More affectation after LASEK \\
\hline$(\mathrm{Al}-\mathrm{Nashar}$ et al. 2017) & ORA & SMILE; PRK & No differences among techniques \\
\hline \multicolumn{4}{|l|}{ TECHNIQUE VARIATIONS } \\
\hline$(\text { Kamiya et al. 2014) })^{77}$ & ORA & SMILE, FLEx & $\begin{array}{l}\text { The presence or absence of flap } \\
\text { lifting does not significantly affect } \\
\text { biomechanical parameters. }\end{array}$ \\
\hline$\left(\right.$ Shen et al. 2014) ${ }^{78}$ & CORVIS & SMILE & $\begin{array}{l}\text { Differences pre/post due to the } \\
\text { extracction of the lenticule and not to } \\
\text { its sculpting }\end{array}$ \\
\hline (Mastropasqua et al. 2014) $)^{79}$ & CORVIS & SMILE & $\begin{array}{l}\text { No significant modifications in } \\
\text { biomechanical properties were } \\
\text { observed after SMILE }\end{array}$ \\
\hline$(\text { El-Massry et al. 2015) })^{80}$ & ORA & SMILE & $\begin{array}{l}\text { Less biomechanical affectation with } \\
160 \mu \mathrm{m} \text { compared to } 100 \mu \mathrm{m}\end{array}$ \\
\hline$(\text { Leccisotti et al. 2016) })^{81}$ & CORVIS & FS-LASIK & $\begin{array}{l}\text { There exists changes in } \\
\text { biomechanical properties after } \\
\text { femtosecond creation of a LASIK flap }\end{array}$ \\
\hline$(\text { Fernandez et al. 2017) })^{82}$ & CORVIS & SMILE & $\begin{array}{l}\text { No differences among low, medium } \\
\text { and high myopia after consideration } \\
\text { of removed central corneal thickness }\end{array}$ \\
\hline
\end{tabular}

SMILE: Small Incision Lenticule Extraction,; FLEx: Femtosecond Lenticule Extraction; LASEK: Laser-Assisted Subepithelial Keratomileusis; LASIK: Laser assisted in Situ Keratomileusis (microkeratome flap); FS-LASIK: Laser assisted in Situ Keratomileusis (femtosecond flap); FS-LASIK WF: Femtosecond Laser assisted in Situ Keratomileusis Wavefront Guided; ORA: Ocular Response Analyzer (Reichert Ophthalmic Instruments, Depew, NY, USA); CORVIS: Corvis ST (Oculus Optikgeräte GmbH, Wetzlar, Germany). 